\title{
Evaluación de la capacidad auditiva en estudiantes de una facultad de odontología en Colombia
}

\section{Avaliação da capacidade auditiva em estudantes de uma faculdade de dentistria na Colômbia}

\section{Assessment of hearing capacity in students of a faculty of dentistry in Colombia}

Karol Yurley Cárdenas-Suárez ${ }^{1 凶} \underline{\mathrm{ORCID}}$, Leomaris Perales-Contreras ${ }^{1 凶} \underline{\mathrm{ORCID}}$, Andrés David GaleanoOrtiz ${ }^{1 凶} \underline{\mathrm{ORCID}}$, Andrea Johanna Almario-Barrera ${ }^{2 凶} \underline{\mathrm{ORCID}}$, Yuli Alexandra Ruiz-Orejarena ${ }^{3 凶} \underline{\mathrm{ORCID}}$, Angie Melissa Soler-Guarin ${ }^{3 凶} \underline{\mathrm{ORCID}}$, Adriana María Valderrama Carrasco ${ }^{4 凶} \underline{\mathrm{ORCID}}$, Sahira Gimena Franco-Hernández $5 \underline{\square \mathrm{ORCI}}$, Yeny Zulay Castellanos-Domínguez ${ }^{6 凶} \underline{\mathrm{ORCID}}$

${ }^{1}$ Estudiante Facultad de Odontología, Universidad Santo Tomás seccional Bucaramanga-Colombia.

2 Odontóloga Universidad Santo Tomás. Magister en Odontología Universidad Santo Tomás. Docente Facultad de Odontología, Universidad Santo Tomás. Colombia.

${ }^{3}$ Estudiante Programa Fonoaudiología Universidad de Santander.

${ }^{4}$ Magister en Terapia auditivo verbal. Docente programa de Fonoaudiología Universidad de Santander-Colombia.

${ }^{5}$ Magister en Epidemiología. Docente Programa de Fonoaudiología, Universidad de Santander-Colombia.

${ }^{6}$ Magister en Epidemiología. Docente Facultad de Odontología, Universidad Santo Tomás seccional Bucaramanga- Colombia.

Fecha correspondencia: Recibido: mayo de 2020. Aprobado: noviembre de 2021

Forma de citar:

Cárdenas-Suárez KY, PeralesContreras L, Galeano-Ortiz AD, Almario-Barrera AJ, RuizOrejarena YA, Soler-Guarin AM, Valderrama-Carrasco AM, FrancoHernández SG, CastellanosDomínguez YZ. Evaluación de la capacidad auditiva en estudiantes de una facultad de odontología en Colombia. Rev. CES Odont 2021; 34(2): 46-60. https://doi.org/10.21615/ cesodon.5657

$\underline{\text { Open access }}$

\section{Resumen}

Introducción y objetivo: La pérdida de capacidad auditiva por exposición a ruido en ambiente laboral es un problema de salud pública que se constituye en la tercera causa de incapacidad mundial por exposición laboral. En el área de odontología, personal en proceso de formación se ve expuesto al ruido del instrumental usado en los procedimientos clínicos lo que puede conducir a pérdida auditiva inducida por ruido. Evaluar la capacidad auditiva de estudiantes de una facultad de odontología en Colombia. Materiales y métodos: Se realizó un estudio observacional analítico de corte transversal, en estudiantes de odontología de sexto a décimo semestre; se hizo encuesta autodiligenciada y valoración clínica audiológica a todos los participantes. La base de datos se consolidó en Microsoft Excel y se analizó en el programa estadístico STATA 14.0. El análisis 
(C) Derecho de autor

Licencia creative commons

Ética de publicaciones

Revisión por pares

Gestión por Open Journal

System

DOI: 10.21615/cesodon.5657

ISSNe 2215-9185

ISSN 0120-971X

Publica con nosotros estadístico incluyó descripción de los datos usando media y desviación estándar (datos cuantitativos) y frecuencias absoluta y relativa (datos cualitativos) los cuales se acompañaron de intervalos de confianza del 95\%. El análisis bivariado se realizó con test exacto de Fisher y prueba de Chi cuadrado así como prueba de t de Student y ANOVA. Resultados: Se evidenció una pérdida auditiva de $77,8 \%$ para el oído derecho y el $76,2 \%$ para el oído izquierdo. Conclusión: Los estudiantes de odontología presentaron alteración en su capacidad auditiva. La edad y el semestre académico se asocian con la pérdida de la capacidad auditiva.

Palabras clave: audiometría; pérdida auditiva; facultades de odontología; estudiantes de odontología.

\section{Abstract}

Introduction and objective: Loss of hearing ability due to exposure to noise in the workplace is a public health problem that constitutes the third cause of disability worldwide due to occupational exposure. In the dental area, personnel are exposed to the noise of the instruments used in clinical procedures, which can lead to noise-induced hearing loss. To evaluate the hearing capacity of students from a dental school in Colombia. Materials and methods: An analytical cross-sectional study was carried out in dental students from sixth to tenth semester; the participants' information was obtained through a self-filled survey and clinical audiological evaluation. The database was consolidated in Microsoft Excel and exported to the STATA 14.0 program where the information was processed. The analysis plan included description of the data using mean and standard deviation (quantitative data) and absolute and relative frequencies (qualitative data) which are accompanied by $95 \%$ confidence intervals. Bivariate analysis was performed with Fisher's exact test and Chi square test, as well as Student's t-test and ANOVA. Results: A hearing loss of $77.8 \%$ was evident for the right ear and $76.2 \%$ for the left ear. Conclusion: Dental students presented alteration in their hearing ability. Age and academic semester are associated with loss of hearing ability.

Keywords: audiometry; hearing loss; schools dental; students dental. 


\section{Resumo}

Introdução e objetivo: A perda da capacidade auditiva devido à exposição ao ruído no local de trabalho é um problema de saúde pública que é a terceira principal causa de incapacidade no mundo devido à exposição ocupacional. Na área de odontologia, o pessoal é exposto ao ruído dos instrumentos utilizados nos procedimentos clínicos, o que pode levar à perda auditiva induzida por ruído. avaliar a capacidade auditiva de estudantes de uma escola de odontologia na Colômbia. Materiais e métodos: Um estudo transversal analítico foi realizado em estudantes de odontologia do sexto ao décimo semestre; as informações dos participantes foram obtidas por meio de inquérito auto-preenchido e avaliação audiológica clínica. O banco de dados foi consolidado no Microsoft Excel ${ }^{\circledR}$ e exportado para o programa STATA 14.0, onde as informações foram processadas. O plano de análise incluiu a descrição dos dados usando média e desvio padrão (dados quantitativos) e frequências absolutas e relativas (dados qualitativos) acompanhadas por intervalos de confiança de $95 \%$. A análise bivariada foi realizada com o teste exato de Fisher e qui-quadrado, bem como o teste t de Student e ANOVA. Resultados: Perda auditiva de na orelha direita foi $77,8 \%$ na orelha direita e $76,2 \%$ na orelha esquerda. Conclusão: Os estudantes de odontologia apresentaram alteração na capacidade auditiva. A idade e o semestre acadêmico estão associados à perda da capacidade auditiva.

Palavras chave: audiometria; perda auditiva; faculdades de Odontologia; estudantes de odontologia.

\section{Introducción}

La pérdida de la capacidad auditiva se define como la disminución de la audición mayor a 20 decibeles $(\mathrm{dB})$ en el oído con mejor audición en una persona adulta y corresponde a un fenómeno que ocurre entre otras causas, al daño o fatiga en las células ciliadas cocleares ${ }^{(1,2)}$. La Organización Mundial de la Salud (OMS) clasifica la hipoacusia o perdida de la audición de acuerdo a su grado de severidad o afectación como leve (21-40 dB), moderada (41-60 dB), severa (61-80 dB) o profunda ( $81 \mathrm{~dB}$ o más) ${ }^{(3)}$ y estima que $6,1 \%$ de la población mundial vive con esta condición ${ }^{(4)}$.

Esta patología representa un problema de salud para el trabajador que ocupacionalmente se encuentra expuesto a niveles de ruido que superan los 85 decibeles (dB). Alrededor del $5 \%$ de la población mundial sufre de pérdida auditiva inducida por ruido y actualmente es la cuarta causa de años vividos con discapacidad ${ }^{(5,6)}$. Anualmente 22 millones de trabajadores están expuestos a ruidos potencialmente dañinos ${ }^{(2)}$. Si bien no se asocia a muertes si se constituye en la primera causa de discapacidad laboral en el mundo con cerca de 5,8 millones de DALYS ${ }^{(7)}$. 
En el área de la salud, los odontólogos se ven expuestos a un alto riesgo de pérdida auditiva inducida por ruido (DAIR) debido a los altos niveles de ruido producidos a partir del instrumental usado durante los procedimientos odontológicos como la pieza de mano, eyector, turbina, micromotor, entre otros ${ }^{(8-10)}$. Estudios han documentado pérdida auditiva en este grupo de profesionales entre $15 \%$ y $20 \%{ }^{(11-13)}$, así como evidencia sobre la variabilidad en la exposición al nivel de ruido de acuerdo con la especialidad clínica que se ejecute ${ }^{(14-17)}$.

Para el caso de estudiantes de odontología, investigaciones han revelado DAIR del $10 \%{ }^{(18)}$ hasta un $51,4 \%$ de hipoacusia leve y moderada ${ }^{(19)}$, con una amplia variabilidad en los niveles de exposición promedio de acuerdo con la especialidad o tipo de procedimiento ${ }^{(9,14)}$. Un estudio realizado en las clínicas odontológicas de la Universidad de Cartagena en Colombia reportó picos de 79-84 dB, lo que supera los límites de la normativa nacional vigente ${ }^{(20)}$. Así, es posible que los estudiantes desde su proceso de formación profesional estén potencialmente expuestos a niveles de ruido que pongan en riesgo su salud auditiva. Por lo anterior, el objetivo de este estudio fue evaluar la capacidad auditiva de los estudiantes de una facultad de odontología con el fin de determinar si existe o no compromiso auditivo por la exposición a ruido durante su proceso de formación.

\section{Materiales y métodos}

\section{Tipo de estudio}

Se realizó un estudio observacional analítico de corte transversal ${ }^{(21)}$.

\section{Población muestra y muestreo}

La población de este estudio estuvo compuesta por estudiantes de sexto a décimo semestre del segundo período académico del 2019 de la Facultad de Odontología de la Universidad Santo Tomás. La estimación de tamaño de muestra se realizó teniendo en cuenta el trabajo de Montenegro ${ }^{(22)}$ donde se incluyeron 50 odontólogos para estimar la prevalencia de hipoacusia inducida por exposición ocupacional al ruido. Mediante muestreo probabilistico estratificado ${ }^{(23)}$ se identificaron los participantes.

\section{Criterios de selección}

Se incluyeron estudiantes activos de pregrado de odontología para el segundo semestre de 2019, que fueran mayores de edad y que estuvieran cursando las clínicas (sexto a décimo semestre). Fueron excluidos los estudiantes con antecedentes médicos de origen hereditario 
que influyan en el diagnóstico auditivo ocupacional así como aquellos que presentaban tapón de cera al momento de la valoración.

\section{Variables del estudio}

La variable dependiente fue el nivel de audición que se midió mediante una prueba tamiz y una audiometría clínica. Las variables independientes fueron: edad, sexo, procedencia, estado civil y estrato socioeconómico, uso de audífonos con carácter recreacional, uso de protectores, otalgia, otorrea, acúfenos, barotrauma, eccema ótico, entre otros.

\section{Instrumento}

La recolección de datos se llevó a cabo mediante la aplicación de una encuesta estructurada y autodiligenciada a los estudiantes. Se aplicó una prueba piloto con el $10 \%$ de la muestra para verificar y evaluar la suficiencia del instrumento.

\section{Procedimiento}

Los estudiantes seleccionados para hacer parte del estudio fueron abordados en las aulas de clase donde se les informó y se les invitó a participar en el estudio. Quienes aceptaron ingresar en el estudio firmaron consentimiento informado, diligenciaron la encuesta y posteriormente, fueron evaluados por medio de la prueba de otoscopia que dió al evaluador la posibilidad de determinar la integridad de la membrana timpánica; la acumetría con diapasones de 500 hz mediante las pruebas de rinne y webber que permitió considerar un topo diagnóstico en cuanto a una posible lesión y se complementó con la prueba objetiva de obtención del umbral auditivo a través de la audiometría tamiz tomando en cuenta la valoración en vía aérea de forma independiente para cada oído, en las frecuencias de 250, 500, 1000, 2000, 3000, 4000, 6000 y $8000 h z$. Se empleó para la evaluación un audiómetro electrónico portátil con transductores supra aurales y se acondicionó un espacio cerrado con condiciones de silencio máximo posible.

La prueba tamiz realizada permitió obtener datos de evaluación de la capacidad auditiva por vía aérea en las frecuencias de 250 a 8000 hz de cada uno de los participantes independizando la obtención del umbral por cada oído. Los hallazgos obtenidos mediante la prueba tamiz realizada en la evaluación y los datos de la encuesta practicada a cada uno de los participantes del estudio, fueron registradas en el programa Microsoft Excel $₫$, además fueron digitados por duplicado de forma independiente para realizar la validación de la base de datos. Se utilizó el programa estadístico STATA 14 para el análisis. 


\section{Análisis estadístico}

Se presenta análisis univariado, donde se calcularon frecuencias absolutas y porcentajes junto con el intervalo de confianza del 95\% (IC 95\%) para las variables cualitativas y medidas de tendencia central junto con medidas de dispersión (media y desviación estándar o mediana y rango intercuartilico) para las variables cuantitativas, dependiendo de la distribución establecida por la prueba Shapiro Wilk.

El análisis bivariado estableció posibles asociaciones entre la variable dependiente y las variables independientes. Por medio de la prueba $\mathrm{Chi}^{2}$ o la prueba Exacta de Fisher. También, de acuerdo con la distribución de las variables cuantitativas, se realizaron comparaciones con variables cualitativas usando las pruebas t de Student o U de Mann-Whitney y Annova o Kruskall Wallis. Para todas las pruebas se consideró significancia estadística para aquellos valores de $\mathrm{p}<0,05$.

\section{Consideraciones éticas}

Esta investigación se acogió a los principios contemplados en la resolución 08430 de 1993 que establece la normativa científico administrativa para la investigación en salud en Colombia ${ }^{(24)}$. Conforme al artículo 11 de la resolución, el estudio se clasificó como de riesgo mínimo. Así mismo, esta investigación se sometió a revisión por parte del comité de investigaciones de la Universidad Santo Tomás y fue aprobado por el comité de ética acta número 00152019114022019 de 2019.

\section{Resultados}

Un total de 63 estudiantes participaron en el estudio. De éstos el 84,1\% eran mujeres y 95,2\% estaban solteros; la mediana de edad fue 21 años con un rango intercuartil de 21 a 22 años. Respecto al uso de audífonos, el $68,3 \%$ reportó su uso y tan solo el 1,6\% manifestó usar protectores auditivos con una frecuencia de una vez por semana (Tabla 1). 
Tabla 1. Distribución de las características sociodemográficas de los participantes.

\begin{tabular}{|c|c|c|c|}
\hline Variable & Categoría & $n(\%)$ & IC 95\% \\
\hline \multirow{2}{*}{ Sexo } & Femenino & $53(84,1)$ & $72,7-92,1$ \\
\hline & Masculino & $10(15,9)$ & $7,9-27,3$ \\
\hline \multirow{2}{*}{ Estado civil } & Soltero & $60(95,2)$ & $86,7-99,0$ \\
\hline & Unión libre & $3(4,8)$ & $1,0-13,3$ \\
\hline \multirow{4}{*}{ Semestre académico cursado } & $\mathrm{VI}$ & $10(15,9)$ & $7,9-27,3$ \\
\hline & VII & $14(22,2)$ & $12,7-34,4$ \\
\hline & VIII & $18(28,6)$ & $17,9-41,3$ \\
\hline & IX & $21(33,3)$ & $22,0-46,3$ \\
\hline \multirow{5}{*}{ Estrato socio económico } & Dos & $2(3,2)$ & $0,4-11,0$ \\
\hline & Tres & $24(38,1)$ & $26,1-51,2$ \\
\hline & Cuatro & $26(41,3)$ & $29,0-54,4$ \\
\hline & Cinco & $10(15,9)$ & $7,9-27,3$ \\
\hline & Seis & $1(1,6)$ & $0,04-8,5$ \\
\hline \multirow{2}{*}{ Uso de audífonos } & $\mathrm{Si}$ & $43(68,3)$ & $55,3-79,4$ \\
\hline & No & $20(31,8)$ & $20,6-44,7$ \\
\hline \multirow{3}{*}{ Frecuencia de uso de audífonos } & Una vez por semana & $13(30,3)$ & $17,2-46,1$ \\
\hline & 2-3 veces por semana & $15(34,9)$ & $21,0-50,9$ \\
\hline & 4-7 veces por semana & $15(34,9)$ & $21,0-50,9$ \\
\hline \multirow{2}{*}{ Uso de protectores auditivos } & $\mathrm{Si}$ & $1(1,6)$ & $0,04-8,5$ \\
\hline & No & $62(98,4)$ & $91,5-99,9$ \\
\hline
\end{tabular}

En cuanto a los hallazgos relacionados con daño del oído se resalta la presencia de acúfenos $(31,8 \%)$ y sensación de plenitud aural $(55,6 \%)$ (Tabla 2$)$.

Tabla 2. Hallazgos relacionados con anormalidades identificadas en oído.

\begin{tabular}{lcc}
\hline Hallazgo & $\boldsymbol{n ( \% )}$ & $\boldsymbol{I C 9 5 \%}$ \\
\hline Otalgia & $21(33,3)$ & $21,9-46,3$ \\
\hline Sensación de plenitud aural & $35(55,6)$ & $42,5-68,1$ \\
\hline Eccema ótico & $8(12,7)$ & $5,6-23,5$ \\
\hline Acúfenos & $20(31,8)$ & $20,6-44,7$ \\
\hline Otitis & $8(12,7)$ & $5,6-23,5$ \\
\hline Tinnitus & $13(20,6)$ & $11,5-32,7$ \\
\hline Vértigo & $17(27,0)$ & $16,6-39,7$ \\
\hline Siente que escucha menos & $22(34,9)$ & $23,3-48,0$ \\
\hline
\end{tabular}


Respecto a la evaluación de la pérdida de capacidad auditiva por audiometría se encontró que el $22,2 \%$ (oído derecho) y $23,8 \%$ (oído izquierdo) de los participantes tenían una capacidad auditiva normal. No se reportaron pérdidas auditivas severas ni profundas, sin embargo el $77,8 \%$ y el $76,2 \%$ reportaron una pérdida de capacidad auditiva leve y moderada en oído derecho e izquierdo respectivamente (Tabla 3 ).

Tabla 3. Resultados de la audiometría oído derecho y oído izquierdo.

\begin{tabular}{lcc}
\hline Capacidad auditiva & $\begin{array}{c}\text { Oído derecho } \\
\boldsymbol{n}(\%)\end{array}$ & $\begin{array}{c}\text { Oído izquierdo } \\
\boldsymbol{n}(\%)\end{array}$ \\
\hline Normal & $14(22,2)$ & $15(23,81)$ \\
\hline Leve & $48(76,2)$ & $45(71,4)$ \\
\hline Moderada & $1(1,6)$ & $3(4,8)$ \\
\hline
\end{tabular}

Se realizó un análisis bivariado para identificar potenciales variables relacionadas con la pérdida de capacidad auditiva en los estudiantes. Este análisis identificó que tanto la edad del estudiante como el semestre académico cursado se asocian con la pérdida de capacidad auditiva $(p<0,05)$. Para el caso de las variables clínicas no se encontró asociación con ninguna de éstas (Tabla 4). 
Tabla 4. Distribución de la pérdida auditiva de los estudiantes según las variables sociodemográficas y clínicas para cada oído.

\begin{tabular}{|c|c|c|c|c|c|c|c|}
\hline \multirow[b]{3}{*}{ Variable } & \multirow[b]{3}{*}{ Categoría } & \multicolumn{5}{|c|}{ Pérdida auditiva } & \multirow[b]{3}{*}{ Valor } \\
\hline & & \multicolumn{2}{|c|}{ Oído derecho } & \multicolumn{3}{|c|}{ Oído izquierdo } & \\
\hline & & $\begin{array}{c}\text { no } \\
n(\%)\end{array}$ & $\begin{array}{c}s i \\
n(\%)\end{array}$ & Valor $p$ & $\begin{array}{c}\text { no } \\
n(\%)\end{array}$ & $\begin{array}{c}s i \\
n(\%)\end{array}$ & \\
\hline \multirow{2}{*}{ Sexo } & Femenino & $10(18,9)$ & $43(81,1)$ & \multirow{2}{*}{0,209} & $11(20,8)$ & $4(79,3)$ & \multirow{2}{*}{0,231} \\
\hline & Masculino & $4(40)$ & $6(60)$ & & $4(40)$ & $6(60)$ & \\
\hline Edad & $\dot{X} \pm D E$ & $21,2 \pm 3,2$ & $21,9 \pm 1,9$ & $0,009 *$ & $21,2 \pm 3,0$ & $21,9 \pm 2,0$ & $0,029 *$ \\
\hline \multirow{2}{*}{$\begin{array}{l}\text { Semestre } \\
\text { académico }\end{array}$} & VI-VII & $13(30,9)$ & $29(69,1)$ & \multirow{2}{*}{$0,024 *$} & $15(35,7)$ & $27(64,3)$ & \multirow{2}{*}{$0,001^{*}$} \\
\hline & VIII-IX & $1(4,8)$ & $20(95,2)$ & & $0(0)$ & $21(100)$ & \\
\hline \multirow{2}{*}{ Procedencia } & Urbana & $12(20)$ & $48(80)$ & \multirow{2}{*}{0,121} & $14(23,3)$ & $46(75,7)$ & \multirow{2}{*}{0,564} \\
\hline & Rural & $2(66,7)$ & $1(33,3)$ & & $1(33,3)$ & $2(66,7)$ & \\
\hline \multirow{2}{*}{$\begin{array}{l}\text { Estrato } \\
\text { socioeconómico }\end{array}$} & $1-3$ & $6(23,1)$ & $20(76,9)$ & \multirow{2}{*}{0,891} & $9(34,6)$ & $17(65,4)$ & \multirow{2}{*}{0,091} \\
\hline & $4-6$ & $8(21,6)$ & $29(78,4)$ & & $6(16,2)$ & $31(83,8)$ & \\
\hline \multirow{2}{*}{$\begin{array}{l}\text { Uso de } \\
\text { audífonos }\end{array}$} & No & $3(15)$ & $17(85)$ & \multirow{2}{*}{0,518} & $3(15)$ & $17(85)$ & \multirow{2}{*}{0,349} \\
\hline & Sí & $11(25,6)$ & $32(74,4)$ & & $12(27,9)$ & $31(72,1)$ & \\
\hline \multirow{2}{*}{ Otalgia } & No & $11(26,2)$ & $31(73,8)$ & \multirow{2}{*}{0,350} & $12(28,6)$ & $30(71,4)$ & \multirow{2}{*}{0,347} \\
\hline & Sí & $3(14,3)$ & $18(85,7)$ & & $3(14,3)$ & $18(85,7)$ & \\
\hline \multirow{2}{*}{ Eccema ótico } & No & $12(21,8)$ & $43(78,2)$ & \multirow{2}{*}{1,000} & $12(21,8)$ & $43(78,2)$ & \multirow{2}{*}{0,382} \\
\hline & Sí & $2(25)$ & $6(75)$ & & $3(37,5)$ & $5(76,2)$ & \\
\hline \multirow{2}{*}{ Acúfenos } & No & $9(20,9)$ & $34(79,1)$ & \multirow{2}{*}{0,718} & $11(25,6)$ & $32(74,4)$ & \multirow{2}{*}{0757} \\
\hline & Sí & $5(25)$ & $15(75)$ & & $4(20)$ & $16(80)$ & \\
\hline Sensación de & No & $8(28,6)$ & $20(71,4)$ & & $10(35,7)$ & $18(64,3)$ & \\
\hline plenitud aural & Sí & $6(17,1)$ & $29(82,9)$ & $0,2 / 8$ & $5(14,3)$ & $30(85,7)$ & $0,0 / 3$ \\
\hline Otitis & No & $13(23,6)$ & $42(76,4)$ & 0671 & $14(25,4)$ & $41(74,6)$ & 0667 \\
\hline Utilis & Sí & $1(12,5)$ & $7(87,5)$ & $0,0 / 1$ & $1(12,5)$ & $7(87,5)$ & 0,007 \\
\hline Tinnituc & No & $12(24)$ & $38(76)$ & O 71 & $12(24)$ & $38(76)$ & 10 \\
\hline 111micus & Sí & $2(15,4)$ & $11(84,6)$ & 0,114 & $3(23,1)$ & $10(76,9)$ & 1,000 \\
\hline & No & $11(23,9)$ & $35(76,1)$ & & $9(19,6)$ & $37(80,4)$ & 0192 \\
\hline vertigo & Sí & $3(17,7)$ & $14(82,4)$ & $0, / 41$ & $6(35,3)$ & $11(64,7)$ & 0,193 \\
\hline Siente que & No & $9(21,9)$ & $32(78,1)$ & O ०४4 & $10(24,4)$ & $31(75,6)$ & 0883 \\
\hline escucha bien & Sí & $5(22,7)$ & $17(77,3)$ & 0,944 & $5(22,7)$ & $17(77,3)$ & 0,883 \\
\hline
\end{tabular}

\section{Discusión}

El presente estudio determinó en estudiantes de odontología una pérdida de capacidad auditiva de $77,8 \%$ y $76,2 \%$ en oído derecho e izquierdo respectivamente, siendo esta pérdida de carácter leve en la mayoría de los casos (76,2\% para el oído derecho y $71,4 \%$ para el oído izquierdo). No se reportaron pérdidas auditivas de carácter severo o profundo. Estos resultados son similares a los reportados por Rivera $\mathrm{YL}$ y colaboradores donde se estimó una pérdida auditiva inducida en docentes de clínicas odontológicas de 62,9\% para el oído izquierdo y de 
$88,9 \%$ en el oído derecho. Estos resultados son ligeramente superiores a los encontrados en este estudio lo cual puede deberse a la edad del grupo poblacional evaluado que presentan más años de práctica profesional y por ende mayor tiempo de exposición al ruido laboral ${ }^{(25)}$.

Dentro de las variables sociodemográficas se encontró que más del $80 \%$ de los participantes eran de sexo femenino y con una edad promedio de 21 años lo que es consistente con lo reportado por Micin-Carvallo y colaboradores quienes encontraron que en el perfil de estudiantes de Chile que ingresan a carreras del área de la salud para el año 2017 eran en su mayoría mujeres $(72,4 \%)$ y la edad promedio de ingreso es 19,3 años ${ }^{(26)}$.

Hernández $\mathrm{S}$ y colaboradores refieren que la pérdida auditiva inducida por ruido causada por la exposición prolongada durante varios años a ruido intenso es una condición que interfiere en la comunicación, así como en la integración social y la calidad de vida de las personas que la padecen. Para el caso del ambiente laboral el personal se ve expuesto al ruido externo del ambiente de trabajo (27); en el caso del profesional de odontología se suma el ruido generado del equipo de trabajo como amalgamadores, compresores y eyectores, entre otros, así como la marca de la pieza de mano ${ }^{(20,28,29)}$. Así, estudios han encontrado pérdida de la capacidad auditiva mayor estadísticamente significativa en odontólogos comparado con un grupo control ${ }^{(11)}$.

El presente trabajo encontró asociación entre la pérdida de la capacidad auditiva con la edad y el semestre académico cursado por los estudiantes. Este hallazgo es consistente con lo descrito por Ahmed y colaboradores quienes encontraron mayor frecuencia de problemas de audición para estudiantes de odontología de segundo a quinto semestre en comparación con los de primer semestre ${ }^{(18)}$. Al compararse los odontólogos con población general, el tiempo de exposición laboral al ruido así como la edad se han identificado como variables asociadas a la pérdida de la capacidad auditiva ${ }^{(13)}$. Así mismo Al - Rawi y colaboradores evidenciaron que la edad y los años de experiencia de los odontólogos mostraron una correlación positiva con una capacidad auditiva reducida ${ }^{(30)}$.

Un estudio realizado en egresados y estudiantes de odontología de la universidad de Chile no encontró correlación entre los niveles de ruido y la pérdida auditiva, no obstante se estableció que a mayor tiempo de exposición menor es la amplitud de la frecuencia 4000 en otoemisiones acústicas ${ }^{(10)}$. 
Le Prell y colaboradores encontraron que el uso del reproductor de música es un factor de riesgo para la pérdida de la audición en los estudiantes universitarios por lo que enfatizan en la importancia de la educación sobre factores de riesgo para la pérdida auditiva teniendo en cuenta que en este grupo poblacional los cambios tempranos en la audición no son fácilmente detectados ${ }^{(31)}$. No obstante, en el presente estudio, no se encontró asociación entre el uso de audífonos y la capacidad auditiva disminuida. Esta discrepancia podría darse por la estrategia de selección y contacto de participantes así como por el instrumento aplicado.

Este estudio usó la audiometría tamiz para hacer la medición de la capacidad auditiva y la otoscopia para la valoración de la permeabilidad de los conductos auditivos externos. De acuerdo con evaluaciones de confiabilidad, la calidad diagnóstica de la audiometría tamiz permite que pueda ser usada como prueba para establecer el grado de deterioro o compromiso auditivo específicamente en la vía aérea determinando así el grado de severidad, no se logra establecer el compromiso en cuanto al diagnóstico el cual se determina con una audiometría clínica complementaria ${ }^{\left({ }^{32}\right)}$. Lo anteriormente descrito podría considerarse como una fortaleza del presente trabajo.

Los datos aquí presentados corresponden a un análisis preliminar realizado con una muestra inicial de estudiantes; por lo anterior, es posible que no se identificaran asociaciones con algunas variables de interés clínico. Así, los hallazgos aquí presentados pueden no mostrar asociaciones estadísticas con algunos factores analizados y reportados previamente en la literatura.

\section{Conclusiones}

Los estudiantes de la facultad de odontología evidencian una marcada pérdida de capacidad auditiva evaluada mediante prueba tamiz de audiometría de 78,5\% para el oído derecho y de $76,2 \%$ para el oído izquierdo. La edad y el semestre cursado se comportaron como variables asociadas a ésta pérdida auditiva.

Este trabajo ha sido financiado por la convocatoria interna XI de la Universidad Santo Tomás seccional Bucaramanga. 


\section{Referencias}

1. Díaz C, Goycoolea M, Cardemil F. Hipoacusia: Trascendencia, Incidencia Y Prevalencia. Rev Médica Clínica Las Condes. 2016;27(6):731-9.

2. Themann $\mathrm{CL}$, Masterson EA, Themann $\mathrm{CL}$, Masterson EA. Occupational noise exposure : A review of its effects, epidemiology, and impact with recommendations for reducing its burden. Acoust Soc Am. 2019;5:3879-905.

3. Graydon K, Waterworth C, Miller H, Gunasekera H. Global burden of hearing impairment and ear disease. J Laryngol Otol. 2019;133(1):18-25.

4. Organización Mundial de la Salud (OMS). Deafness and hearing loss. https://www.who.int/health-topics/hearing-loss. 2019.

5. Oishi N SJ. Emerging treatments for noise-induced hearing loss. Expert Opin Emerg Drugs. 2011;16(2):235-45.

6. The Lancet. Hearing loss: time for sound action. Lancet [Internet]. 2017;390(10111):2414. Disponible en: http://dx.doi.org/10.1016/S0140$\underline{6736(17) 33097-0}$

7. Gakidou E, Afshin A, Abajobir AA, Abate KH, Abbafati C, Abbas KM, et al. Global, regional, and national comparative risk assessment of 84 behavioural, environmental and occupational, and metabolic risks or clusters of risks, 1990-2016: A systematic analysis for the Global Burden of Disease Study 2016. Lancet. 2017;390(10100):1345422.

8. Myers J, John AB, Kimball S FT. Prevalence of Tinnitus and Noise-induced Hearing Loss in Dentists. Noise Health. 2016;18(85):347-54.

9. Theodoroff SM FR. Hearing loss associated with long-term exposure to high-speed dental handpieces. Gent Dent. 2015;63(3):71-6.

10. Espinoza Y, Hernandez K, Ortega G, Pilquil M. Niveles De Ruido Ocupacional Y Desempeño Audiológico En Estudiantes Y Profesionales De Odontología [Internet]. 2013. Disponible en: http://www.tesis.uchile.cl/bitstream/handle/2250/116817/Nivelesderuidoocupacion al y desempeño audiológico en estu.pdf?sequence $=1$ 
11. Alabdulwahhab BM, Alduraiby RI, Ahmed MA, Albatli LI, Alhumain MS, Softah NA, et al. Hearing loss and its association with occupational noise exposure among Saudi dentists: a cross-sectional study. BDJ Open [Internet]. 2016;2(1):16006. Disponible en: http://www.nature.com/articles/bdjopen20166

12. Lazar A, Kauer R RD. Hearing Difficulties Among Experienced Dental Hygienists: A Survey. J Dent Hyg. 2015;89(6):378-83.

13. Khaimook W, Suksamae P, Thitiworn C, Satit C, Ratchada T. The prevalence of noiseinduced occupational hearing loss in dentistry personnel. Work Heal Saf. 2014;62(9):357-60.

14. Burk A. An exploratory study of noise exposures in educational and private dental clinics. J Occup Env Hyg. 2017;13(10):741-9.

15. Grass-Martinez Y, Castañeda-Deroncelé M, Pérez-Sánchez G, Rosell-Valdenebro L R-SL. El ruido en el ambiente laboral estomatológico. Medisan. 2017;21(5):527-33.

16. Gurbuz MK, Çatli T, Cingi C, Yaz A, Bal C. Occupational safety threats among dental personnel and related risk factors. J Craniofac Surg. 2013;24(6):2011-4.

17. Choosong T, Kaimook W, Tantisarasart R, Sooksamear P, Chayaphum S, Kongkamol C, et al. Noise exposure assessment in a dental school. Saf Health Work. 2011;2(4):34854.

18. Ahmed HO, Ali WJ. Noise levels, noise annoyance, and hearing-related problems in a dental college. Arch Environ Occup Heal. 2017;72(3):159-65.

19. Martínez-Cántaro NY, Ayca-Castro I C-QW. Niveles de audiometría y su relación con el ruido ocupacional en estudiantes de la escuela profesional de odontología de la universidad Nacional Jorge Basadre Grohmann en Tacna. Rev Médica Basadrina. 2018;2:21-7.

20. Espinosa JC, Espinosa JC, Julio SO, Cabeza GT, Martínez FG. Niveles De Ruido En Clínicas Odontológicas De La Universidad De Cartagena. Rev Colomb Investig en Odontol [Internet]. 2016;6(17):69-76. Disponible en: https://www.rcio.org/index.php/rcio/article/view/200 
21. Donis H. Tipos de diseños de los estudios clínicos y epidemiológicos. Portal Rev Univ los Andes [Internet]. 2013;2(2):76-99. Disponible en: http://150.185.138.216/index.php/biomedicina/article/download/4410/4200

22. Montenegro JS. Prevalencia y severidad de la hipoacusia inducida por exposición ocupacional al ruido en odontólogos del cercado de Arequipa. 2014.

23. Otzen T, Manterola C. Técnicas de Muestreo sobre una Población a Estudio. Int J Morphol. 2017;35(1):227-32.

24. Ministerio de Salud. Resolución 8430 de 1993. Minist Salud y Protección Soc República Colomb. 1993;1993(Octubre 4):1-19.

25. Rivera Y, Rueda S, Concha S. Pérdida auditiva inducida por ruido evaluada en odontólogos docentes de las clínicas odontológicas de la universidad santo tomas 1. USTASALUD. 2008;6:96-103.

26. Micin Carvallo S, Carreño Mendoza B, Urzúa Martínez S. Perfil de ingreso de estudiantes en carreras del área de la salud. Educ Médica Super [Internet]. 2017 [citado 31 de marzo de 2020];31(3):155-67. Disponible en: http://scielo.sld.cu/scielo.php?script=sci arttext\&pid=S0864$\underline{21412017000300016 \& \operatorname{lng}=e s \& n r m=i s o \& t \operatorname{lng}=e s}$

27. Hernández-Sanchez H G-CM. Hipoacusia inducida por ruido: estado actual. Rev Cub Med Mil. 2006;35(4).

28. Zubick HH, Tolentino AT, Boffa J. Hearing loss and the high speed dental handpiece. Am J Public Health. 1980;70(6):633-5.

29. Singh S, Gambhir RS, Singh G, Sharma S, Kaur A. Noise levels in a dental teaching institute - A matter of concern! J Clin Exp Dent. 2012;4(3):2-6.

30. Al-Rawi NH, Al Nuaimi AS, Sadiqi A, Azaiah E, Ezzeddine D, Ghunaim Q, et al. Occupational noise-induced hearing loss among dental professionals. Quintessence Int [Internet]. 2019;50(3):245-50. Disponible en: http://www.ncbi.nlm.nih.gov/pubmed/30773576

31. Le Prell CG, Hensley BN, Campbell KCM, Hall JW, Guire K. Evidence of hearing loss in a «normally-hearing» college-student population. Int J Audiol. 2011;50(SUPPL. 1):1-29. 
32. Kaf WA, Durrant JD, Sabo DL, Boston JR, Taubman LB, Kovacyk K. Validity and accuracy of electric response audiometry using the auditory steady-state response: Evaluation in an empirical design. Int J Audiol. 2006;45(4):211-23. 